\title{
SUPPORTING INFORMATION \\ Identification of dual-target compounds with antifungal and anti-NLRP3 inflammasome activity
}

David J Lowes ${ }^{1}$, Jian Miao $^{2}$, Rand A Al-waqfi², Kristiana A. Avad ${ }^{2,3}$, Kirk E Hevener ${ }^{4}$, Brian M Peters $^{1,5^{*}}$

1Department of Clinical Pharmacy and Translational Science, College of Pharmacy, University of Tennessee Health Science Center, Memphis, TN 38163

${ }^{2}$ Graduate Program in Pharmaceutical Sciences, College of Graduate Health Sciences, University of Tennessee Health Science Center, Memphis, TN 38163

${ }^{3}$ Doctor of Pharmacy Program, College of Pharmacy, University of Tennessee Health Science Center, Memphis, TN 38163

${ }^{4}$ Department of Pharmaceutical Sciences, College of Pharmacy, University of Tennessee Health Science Center, Memphis, TN 38163

${ }^{5}$ Department of Microbiology, Immunology, and Biochemistry, College of Medicine, University of Tennessee Health Science Center, Memphis, TN 38163

*Address correspondence to Brian M Peters at brian.peters@uthsc.edu or Kirk E Hevener at khevener@uthsc.edu. 


\section{$\underline{\text { Table of Contents }}$}

Table S1. Maybridge Compounds.

Table S2. Strains used or created in this study.

Table S3. Primers used in this study.

Figure S1. IL-1 $\beta$ release in THP-1 cells is largely NLRP3-dependent.

Figure S2. Alignment of AHAS orthologs from several Candida species.

Figure S3. LC/MS confirmation of purity/identify of lead compounds 10 and 10a.

Supporting PDB structures submitted as separate attachments

References 
Table S1. Maybridge Compounds. Details on predicted actives compounds ordered and experimentally tested.

\begin{tabular}{|c|c|c|c|c|c|c|c|}
\hline Cmpd \# & Structure & SMILES & Vendor \# & $\begin{array}{l}\text { AHAS } \\
\text { Docking } \\
\text { Score }\end{array}$ & $\begin{array}{l}\text { NLRP3 } \\
\text { Docking } \\
\text { Score }\end{array}$ & $\begin{array}{l}\text { PAINS } \\
\text { Filter }\end{array}$ & $\begin{array}{l}\text { Aggregation } \\
\text { Filter }\end{array}$ \\
\hline 1 & & $\operatorname{c1cccc}(\operatorname{c1C}([\mathrm{O}-])=0) \operatorname{SccS}(=0)(=0) \operatorname{coc}(\mathrm{Cl}) \operatorname{cc}(\mathrm{Cl}) \operatorname{cc} 2$ & HTS02260 & -9.79 & -8.78 & Passed & Passed \\
\hline 2 & & $\operatorname{c1cccc}(\mathrm{C}([\mathrm{O}-])=\mathrm{O}) \mathrm{c} 1 \mathrm{C}(=\mathrm{O}) \mathrm{N}(\mathrm{C}) \mathrm{CC} 2 \mathrm{ccccn} 2$ & HTS09182 & -10.18 & -8.52 & Passed & Passed \\
\hline 3 & & $\operatorname{COc}(\operatorname{cc} 1) \operatorname{ccc} 1 \mathrm{CCNC}(=\mathrm{O}) \mathrm{c} 2 \mathrm{c}(\mathrm{C}) \mathrm{onc} 2-\mathrm{c} 3 \mathrm{c}(\mathrm{F}) \mathrm{cccc} 3 \mathrm{Cl}$ & JFD01669 & -9.89 & -10.14 & Passed & Passed \\
\hline 4 & & $\mathrm{c} 1 \mathrm{cc}(\mathrm{F}) \mathrm{cc}(\mathrm{F}) \mathrm{c} 1 \mathrm{NC}(=\mathrm{O}) \mathrm{Cn}(\mathrm{n} 2) \mathrm{nnc} 2-\mathrm{c}(\mathrm{cc} 3) \mathrm{ccc} 3 \mathrm{C}$ & NRB00134 & -9.70 & -8.63 & Passed & Passed \\
\hline 5 & & $\mathrm{Cc} 1 \mathrm{c}(\mathrm{Cl}) \mathrm{cc}(\mathrm{cc} 1) \mathrm{NC}(=\mathrm{O}) \mathrm{CC}(\mathrm{CC}([\mathrm{O}-])=\mathrm{O}) \mathrm{c} 2 \mathrm{ccccc} 2$ & PD00456 & -9.47 & -9.17 & Passed & Passed \\
\hline 6 & & $\operatorname{c1ccccc} 1 \mathrm{C}(\mathrm{CC}([\mathrm{O}-])=\mathrm{O}) \mathrm{CC}(=\mathrm{O}) \mathrm{Nc} 2 \operatorname{ccc}(\mathrm{c} 23) \mathrm{CcCC} 3$ & PD00462 & -10.07 & -8.33 & Passed & Passed \\
\hline 7 & & $\operatorname{c1cc}(\mathrm{Cl}) \operatorname{ccc} 1 \mathrm{SC}(\mathrm{C} 2=\mathrm{O}) \mathrm{CC}(=\mathrm{O}) \mathrm{N} 2 \mathrm{c} 3 \mathrm{cc}(\mathrm{Cl}) \mathrm{cc}(\mathrm{Cl}) \mathrm{c} 3$ & RJC03163 & -9.83 & -8.54 & Passed & Passed \\
\hline
\end{tabular}




\begin{tabular}{|c|c|c|c|c|c|c|}
\hline 8 & $\operatorname{c1cc}(\mathrm{F}) \operatorname{ccc} 1 \mathrm{NCC}(\mathrm{O}) \mathrm{Cn} 2 \mathrm{cnc}(\mathrm{c} 23) \mathrm{n}(\mathrm{C}) \mathrm{c}(=\mathrm{O}) \mathrm{n}(\mathrm{C}) \mathrm{c} 3=\mathrm{O}$ & RJC03968 & -9.381 & -9.33 & Passed & Passed \\
\hline 9 & $\mathrm{Clc} 1 \mathrm{cccc}(\mathrm{F}) \mathrm{c} 1 \mathrm{C}(\mathrm{NC} 2=\mathrm{O}) \mathrm{Nc}(\mathrm{c} 23) \operatorname{cccc} 3$ & S12245 & -9.45 & -9.74 & Passed & Passed \\
\hline 10 & $\operatorname{c1} \operatorname{ccccc} 1 \mathrm{C}(=\mathrm{O}) \mathrm{CC}(\mathrm{c} 2 \mathrm{ccc}(\mathrm{F}) \operatorname{cc} 2) \operatorname{Sc}(\mathrm{c} 3 \mathrm{C}([\mathrm{O}-])=\mathrm{O}) \operatorname{cccc} 3$ & S15464 & -10.19 & -9.48 & Passed & Passed \\
\hline 11 & $\mathrm{Fc} 1 \mathrm{c}(\mathrm{Cl}) \mathrm{cc}(\mathrm{cc} 1) \mathrm{SCC}(=\mathrm{O}) \mathrm{c}(\mathrm{c} 2) \mathrm{ccc}(\mathrm{c} 23) \mathrm{OCCO} 3$ & SPB08273 & -9.38 & -8.28 & Passed & Passed \\
\hline 12 & $\mathrm{Oc} 1 \mathrm{cc}(\mathrm{O}) \mathrm{cc}(\mathrm{c} 12) \mathrm{oc}(\mathrm{c}(\mathrm{c} 2=\mathrm{O}) \mathrm{O})-\mathrm{c} 3 \mathrm{cc}(\mathrm{O}) \mathrm{c}(\mathrm{O}) \mathrm{cc} 3$ & XBX00307 & -8.36 & -8.36 & Failed & Passed \\
\hline
\end{tabular}

Structures \& Docking Scores for Known AHAS/NLRP3 Inhibitors

\begin{tabular}{|c|c|c|c|c|c|c|}
\hline 13 & $\operatorname{ccOC}(=0) \operatorname{c} 1 \mathrm{c}(\operatorname{cccc} 1) \mathrm{S}(=\mathrm{O})(=\mathrm{O}) \mathrm{NC}(=\mathrm{O}) \mathrm{Nc}(\mathrm{n} 2) \mathrm{nc}(\mathrm{Cl}) \operatorname{cc} 2 \mathrm{OC}$ & C.E. & -6.06 & -1.98 & Passed & Passed \\
\hline 14 & $\begin{aligned} \mathrm{CC}(\mathrm{C})(\mathrm{O}) \mathrm{c} 1 \mathrm{cc}(\mathrm{oc} 1) \mathrm{S}(=\mathrm{O})(=\mathrm{O}) \mathrm{NC}(=\mathrm{O}) \mathrm{Nc} 2 \mathrm{c}(\mathrm{CCC} 3) \mathrm{c} 3 \mathrm{cc}(\mathrm{c} 24 \\
) \mathrm{CCC} 4\end{aligned}$ & MCC950 & $N / A^{a}$ & -7.64 & Passed & Passed \\
\hline
\end{tabular}

aNo docking results could be generated for MCC950 in AHAS due to steric restrictions of the active site. 
Table S2. Strains used or created in this study.

\begin{tabular}{|c|c|c|c|c|}
\hline Strain Name & Parent & Species & Genotype & Reference \\
\hline SC5314 & $\mathrm{n} / \mathrm{a}$ & C. albicans & reference & 1 \\
\hline [429]0832 & $\mathrm{n} / \mathrm{a}$ & C. auris & wild-type & 1 \\
\hline CD36 & $\mathrm{n} / \mathrm{a}$ & C. dubliniensis & reference & 1 \\
\hline CBS138 & $\mathrm{n} / \mathrm{a}$ & C. glabrata & reference & 1 \\
\hline $81-B-5$ & $\mathrm{n} / \mathrm{a}$ & C. krusei & wild-type & 1 \\
\hline CDC317 & $\mathrm{n} / \mathrm{a}$ & C. parapsilosis & reference & 1 \\
\hline MYA3404 & $\mathrm{n} / \mathrm{a}$ & C. tropicalis & reference & 1 \\
\hline BWP17 & SC5314 & C. albicans & ILV2/ILV2 $\Delta / \Delta \arg 4 \Delta / \Delta$ his1 $\Delta / \Delta$ ura3 & 2 \\
\hline JM01 & BWP17 & C. albicans & ILV2/ $\Delta$ ilv2::ARG4 $\Delta / \Delta$ his1 $\Delta / \Delta$ ura3 & This study \\
\hline JM02 & JM01 & C. albicans & ILV2/Ailv2::ARG4 $\Delta / \Delta h i s 1 \Delta u r a 3 / \Delta u r a 3:: U R A 3$ & This study \\
\hline JM03 & JM01 & C. albicans & ILV2/Ailv2::ARG4 $\Delta / \Delta h i s 1 \Delta u r a 3 / \Delta u r a 3:: U R A 3-P r A C T 1-I L V 2$ & This study \\
\hline JM04 & JM01 & C. albicans & ILV2/Ailv2::ARG4 $\Delta / \Delta h i s 1$ ura3/Dura3::URA3-PrTEF1-ILV2 & This study \\
\hline JM05 & JM02 & C. albicans & ILV2/Dilv2::ARG4 $\Delta / \Delta h i s 1:: H I S 1 \Delta u r a 3 / \Delta u r a 3:: U R A 3$ & This study \\
\hline JM06 & JM03 & C. albicans & 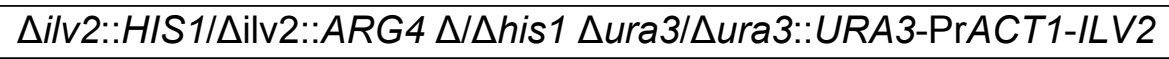 & This study \\
\hline JM07 & JM04 & C. albicans & 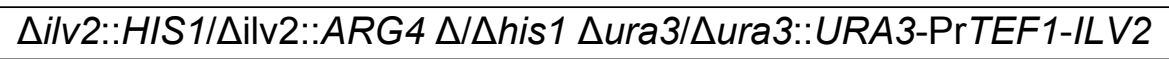 & This study \\
\hline
\end{tabular}


Table S3. Primers used in this study.

\begin{tabular}{|c|c|}
\hline Primer name & Sequence $^{a} 5^{`} \rightarrow 3^{`}$ \\
\hline ACT1PRSEQF & CACCAAGATTTATTGCCAACG \\
\hline TEF1PRSEQF & TTTTTGCTGTTCACTTTCTCG \\
\hline ADH13SEQR & ATATCGCACTCACGTAAACAC \\
\hline ILV2ORF-F-Sall & TCAGTCGACATGATTTCTCGTAATTTGAGAACTTCATCAACG \\
\hline ILV2ORF-R-Mlul & TCAACGCGTCTAATATTTACCACCAGTTCTTTCTTTTC \\
\hline ILV2DISF & TTTGTTTTACTTCTTCTTCTTCTTCTTTCTTCTTTACAGATTCAAACTATTAATTCAAACAAATGTTTTCCCAGTCACGACGTT \\
\hline ILV2DISR & GACTTTTTTTTTTTATAAAAAGAATAATAGATATACAAAACAAGAAGAAGAAGCCAATTTGGTAATGTGGAATTGTGAGCGGATA \\
\hline ILV2AMPF & TCTGTCTAAATGCTACCAACTGGCC \\
\hline ILV2AMPR & ATCACGTCTACTAAATCTTGGACTACGC \\
\hline ARG4INTF & AAGCTAGTGTGGAAAGAAGAG \\
\hline ARG4INTR & AATGACTGAATTATGTCGGTC \\
\hline LUXINTDETF & CTGACCTTTAGTCTTTCCTGC \\
\hline LUXINTDETR & CAGTAGTACTTGTTGTTGTATCG \\
\hline HIS1DETF & TGCATTAATCTTCTTGCCTGC \\
\hline HIS1INTR5 & GGAGGATGAGGAGACAGAAGTTAGT \\
\hline HIS1INTF & ACTGTATCCTCTTCTGTCCCC \\
\hline HIS1INTR & CGACCATATGGGAGAGCTCCC \\
\hline ILV2DETF & GCATTAATGGATGGGGTACCATTAGTGG \\
\hline ILV2DETR & AGCAGCAGATCCATGCATACC \\
\hline ILV2QPCR-R & ATTCTTCTTGGTAATTCCGCCAC \\
\hline CaACT1QPCR-R & TTGGATTCTGGTGATGGTGTTA \\
\hline CaACT1QPCR-R & TCAAGTCTCTACCAGCCAAATC \\
\hline
\end{tabular}

aUnderlined sequences denote complementarity to plasmids pGEMHIS1 and pRSARG4ASpe. Bold, italics sequences denote restriction enzyme sites. 


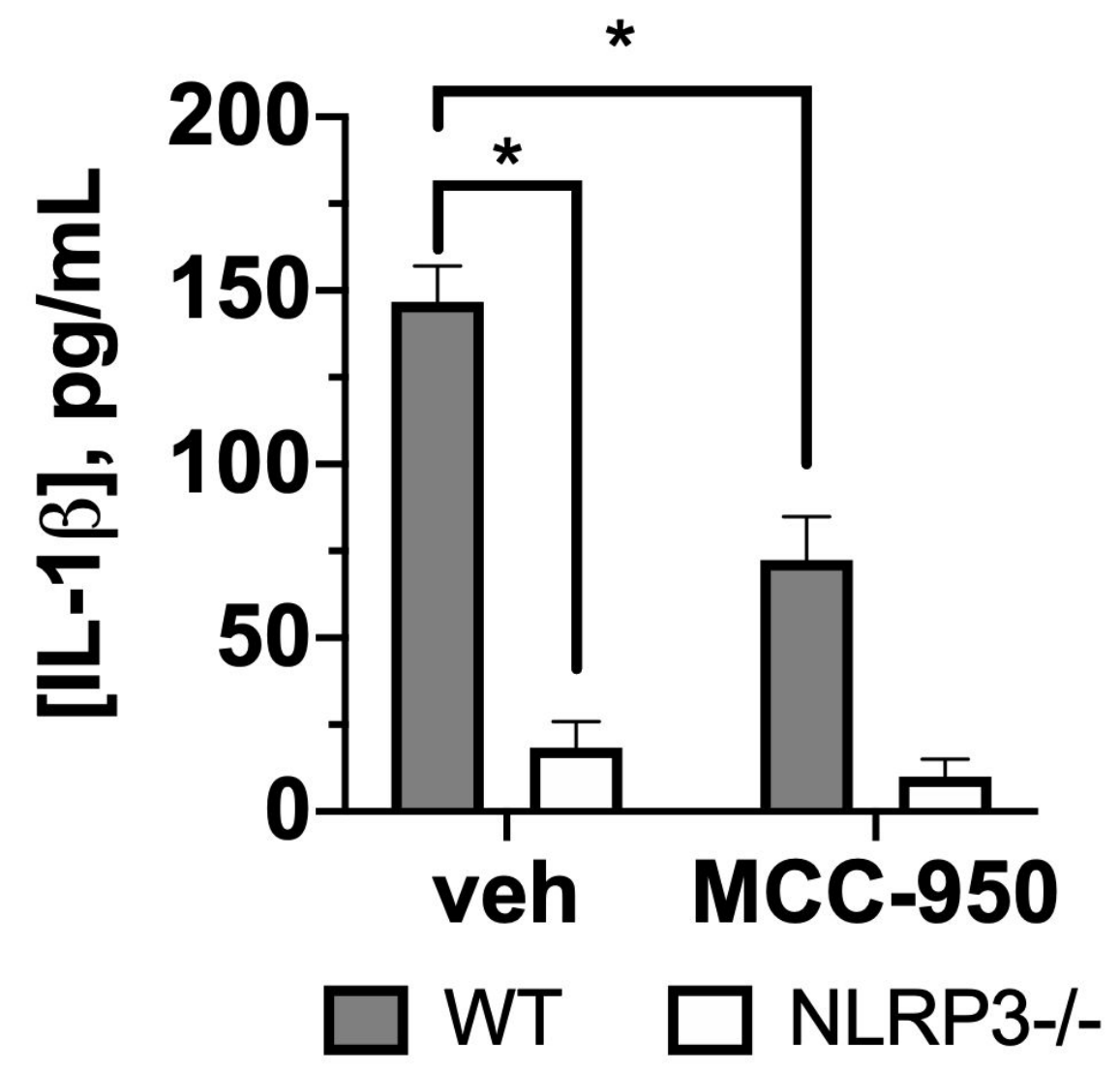

Figure S1. IL-1 $\beta$ release in THP1 cells is largely NLRP3-dependent. Differentiated WT or

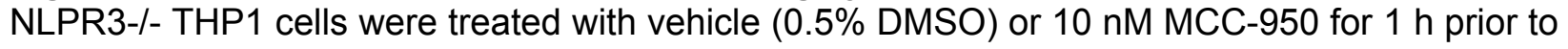
challenge with LPS $(20 \mathrm{ng})$ for $3.5 \mathrm{~h}$ and ATP $(5 \mathrm{mM})$ for $30 \mathrm{~min}$. IL-1 $\beta$ release was quantified by ELISA and blank values subtracted from unstimulated controls. Data is the mean of independent experiments $(n=3)$ conducted in technical quadruplicate. Data was analyzed using a one-way ANOVA and Tukey's post-test. * ${ }^{*} p<0.05$. 

300
310
320
330
340
350

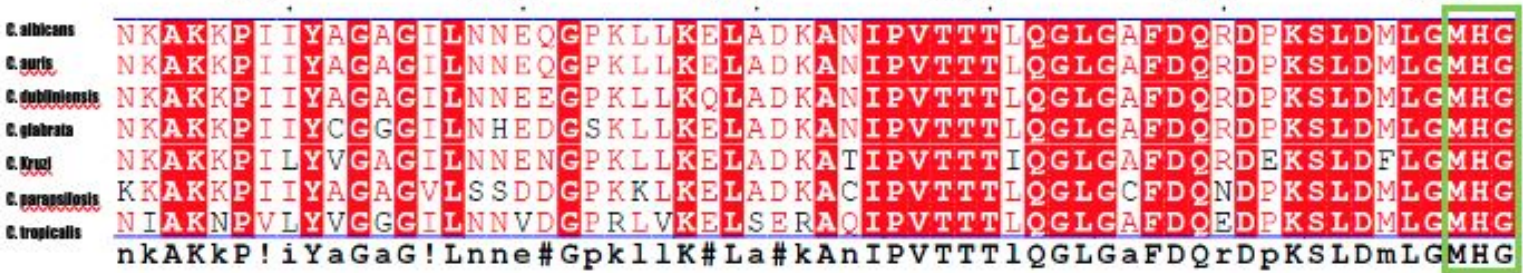

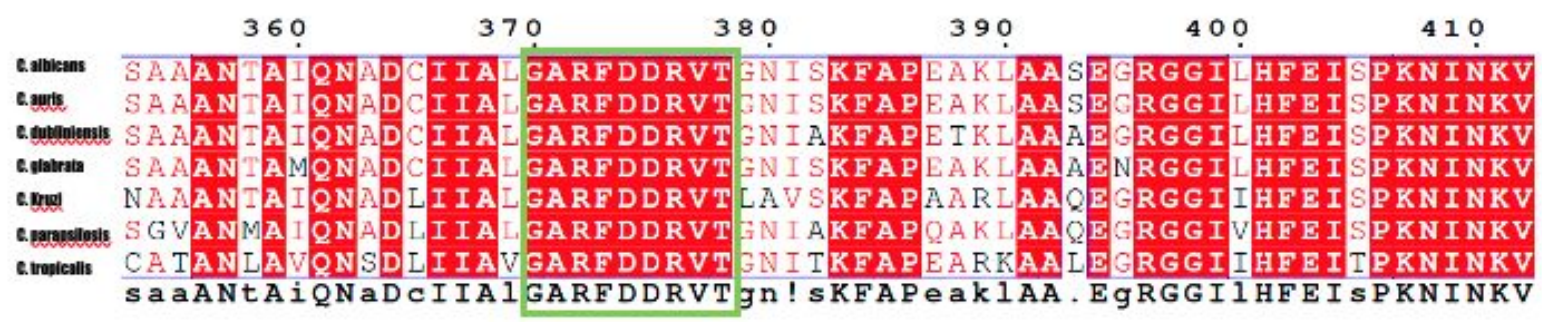

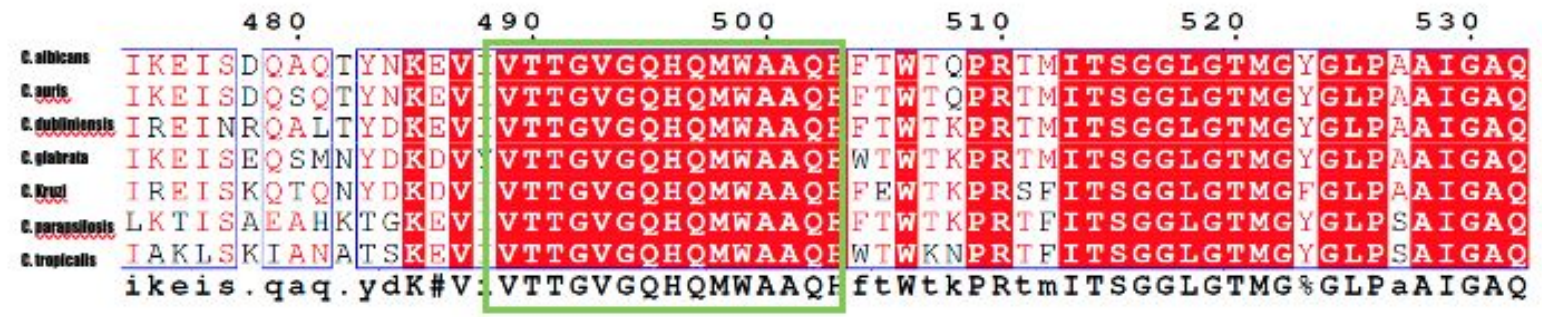

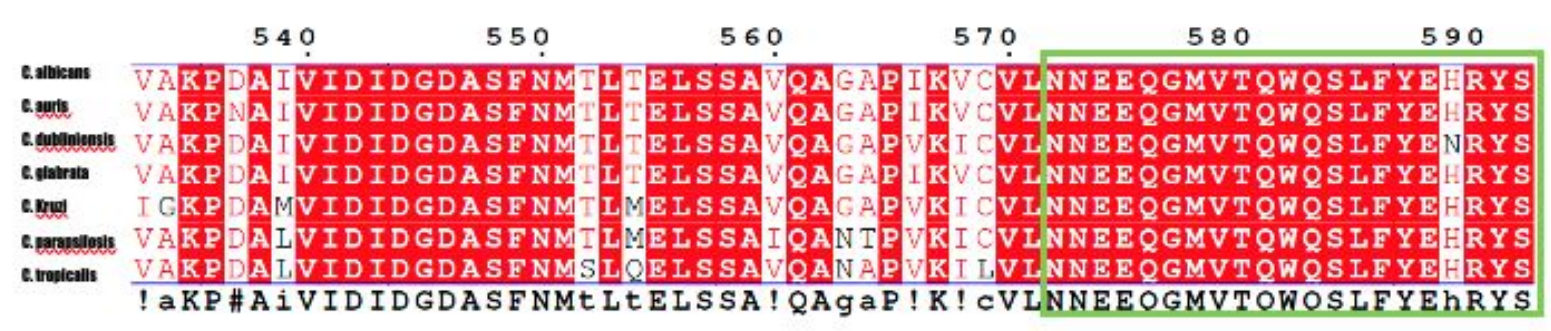

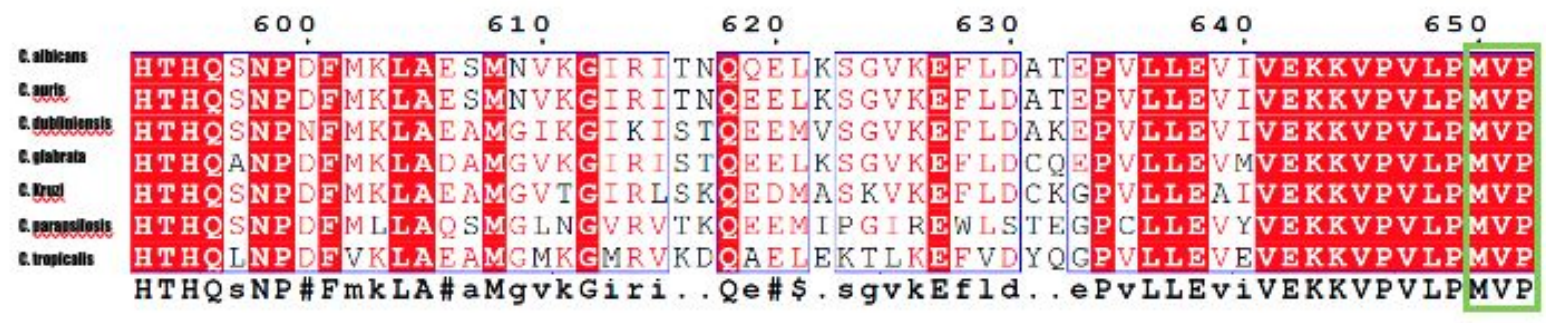

Figure S2. Alignment of AHAS orthologs from several Candida species. ClustalW was used to generate alignments of the AHAS protein from several clinically relevant Candida species and shows a high degree of sequence conservation, with near $100 \%$ identity of active site residues (boxed in green). The sole outlier being $C$. dubliniensis $\mathrm{H} 589 \mathrm{~N}$. 

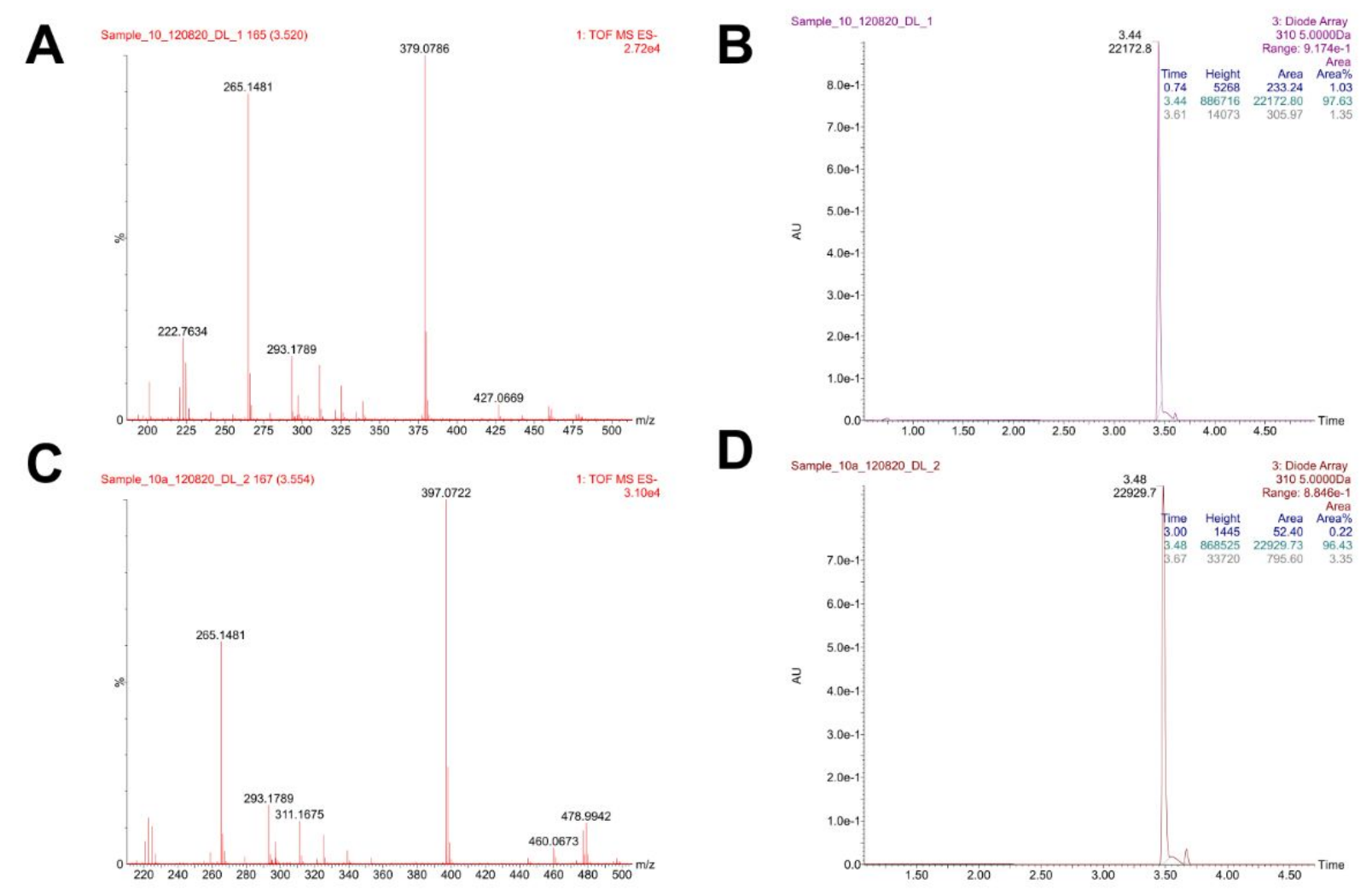

D

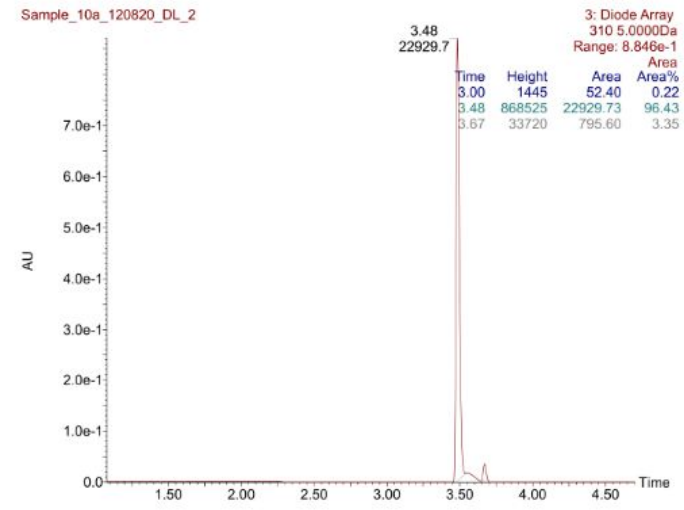

Figure S3. LC/MS confirmation of purity/identify of lead compounds 10 and 10a. (A) Chromatogram of compound 10 using photo diode array detection. (B) Time of flight mass spectrum of compound 10 using negative ionization mode. (C) Chromatogram of compound 10a using photo diode array detection. (D) Time of flight mass spectrum of compound 10a using negative ionization mode. 
Supporting PDB structures submitted as separate attachments:

A. SupportingA_MCC950_NLRP3_CoStructure.pdb

B. SupportingB_Compound10_NLRP3_CoStructure.pdb

C. SupportingC_Compound10_AHAS_CoStructure.pdb 


\section{References}

1. Willems, H. M. E.; Lowes, D. J.; Barker, K. S.; Palmer, G. E.; Peters, B. M. Comparative Analysis of the Capacity of the Candida Species To Elicit Vaginal Immunopathology. Infect Immun 2018, 86, e00527-18.

2. Wilson, R. B.; Davis, D.; Mitchell, A. P. Rapid hypothesis testing with Candida albicans through gene disruption with short homology regions. J Bacteriol 1999, 181, 1868-1874. 